\title{
Estudio Sociolingüístico del Español de Chile (ESECH): recogida y estratificación del corpus de Santiago
}

\author{
Abelardo San Martín Núñez* \\ Silvana Guerrero González \\ Universidad de Chile, Chile
}

\section{INTRODUCCIÓN}

El propósito de este trabajo es presentar los objetivos y la propuesta metodológica del proyecto de Estudio Sociolingüístico del Español de Chile $(\mathrm{ESECH})$, que constituye un grupo de estudio integrado por académicos y estudiantes del Departamento de Lingüística de la Facultad de Filosofía y Humanidades de la Universidad de Chile. El objetivo general del mencionado proyecto es estudiar la influencia de factores sociales y contextuales en el uso lingüístico de la lengua española en Chile, aunque en el estado actual de desarrollo de dicha iniciativa, los miembros del equipo se han concentrado, específicamente, en el análisis de la variación lingüística en el habla capitalina. Para tal cometido, la actividad principal del grupo ha consistido

\footnotetext{
* Para correspondencia, dirigirse a: Abelardo San Martín Núñez (asmartin@uchile.cl),o a Silvana Guerrero González (siguerrero@u.uchile.cl), Departamento de Lingüística, Facultad de Filosofía y Humanidades, Universidad de Chile, Av. Capitán Ignacio Carrera Pinto 1025, tercer piso, Nuñoa, CP 7800284, Santiago, Chile.
} 
en la conformación de un corpus de entrevistas realizadas a una muestra estratificada de sujetos representativos del español hablado en Santiago. En particular, en este trabajo se sintetiza la propuesta del procedimiento de estratificación social de los hablantes santiaguinos que componen la mencionada muestra, la que se basa en la asignación de puntaje, según tres variables demográficas: 1) nivel educacional, 2) profesión u ocupación y 3 ) comuna de residencia. De esta manera, se espera contribuir a un aspecto de la metodología sociolingüística de relativa complejidad y su adecuación a las particularidades de la comunidad lingüística en estudio.

\section{CORPUS ESECH DEL ESPAÑOL HABLADO EN SANTIAGO}

En las investigaciones del grupo ESECH, hasta la fecha, se ha adoptado -en lo principal- la metodología propuesta por Labov (1983) dentro del llamado "paradigma cuantitativo o variacionista" para la descripción sociolingüística del uso de las lenguas en comunidades de habla particulares. Según esta metodología, se recopila un conjunto de entrevistas realizadas a sujetos representativos del habla de dicha comunidad. Luego, se analiza la muestra de entrevistas aplicando procedimientos formales que permiten determinar la existencia de correlaciones entre variables dependientes (fenómenos lingüísticos) y variables independientes (fenómenos socio-demográficos o contextuales). Los fenómenos de variación lingüística que muestran una correlación significativa con factores sociodemográficos o contextuales son tipificados como variables sociolingüísticas. En general, los fundamentos de la metodología aplicada en este tipo de investigaciones aparecen muy bien explicados, en lengua española, tanto en las fuentes metodológicas fundamentales (Moreno Fernández 1990; López Morales 1994 y Hernández Campoy y Almeida 2005), como en los manuales más conocidos de dicha interdisciplina (Moreno Fernández 1998; Silva-Corvalán 2001; López Morales 2004; Blas Arroyo 2005 y Serrano 2011).

El corpus del español hablado en Santiago recogido por el grupo ESECH está conformado por 192 entrevistas sociolingüísticas realizadas a hablantes santiaguinos, entre 2005 y 2012, por los estudiantes en la cátedra de Sociolingüística de los programas de Licenciatura en Lengua y Literatura Hispánicas y Licenciatura en Lengua y Literatura Inglesas de la Universidad de Chile, asignatura dictada por nosotros. Con base en el marco de la metodología variacionista propuesta por Labov, las entrevistas fueron realizadas a hombres y mujeres con características sociodemográficas 
congruentes. En la situación de entrevista, los entrevistadores debían tratar de superar la paradoja del observador ${ }^{1}$ consiguiendo, de esta forma, una muestra significativa de discurso natural grabado (estilo vernáculo ${ }^{2}$ ) de hablantes representativos de la comunidad de habla en estudio. El número de entrevistas antes indicado totaliza, aproximadamente, 186 horas de grabación.

\subsection{CRiterio de SELECCión DE hablantes SANTIAGuinos}

Por lo que refiere a los criterios de asignación de estatus de hablante nativo de Santiago de Chile, se aplicaron las siguientes restricciones en la selección de los sujetos, adaptadas de la propuesta de Prieto (1995-1996: 399):

1. haber nacido y residido en forma ininterrumpida en Santiago,

2. haber nacido y residido en Santiago la mayor parte de sus vidas, salvo por periodos que sumados no superen los cinco años,

3. haber residido en forma ininterrumpida en Santiago desde los cinco años de edad.

1 Según Labov (1983), el objetivo de la investigación lingüística de una comunidad de habla consiste en estudiar la forma en cómo habla la gente cuando no está siendo sistemáticamente observada; sin embargo, solo podemos obtener tales datos mediante la observación sistemática. A esto es a lo que se le ha denominado paradoja del observador. Este dilema se soluciona encontrando la manera de completar la entrevista formal con otros datos o cambiando la estructura de la situación de la entrevista de una u otra manera. En este sentido, una forma de superar esta paradoja consiste en romper las constricciones de la situación de entrevista mediante diversos procedimientos que distraen la atención del sujeto en su propio discurso y pueden hacer brotar su habla más vernácula. Esto puede llevarse a cabo mediante intervalos y cortes definidos de tal manera que el entrevistado suponga inconscientemente que en ese momento no está siendo observado. Asimismo, siguiendo a Labov, también pueden implicarse temas en que los informantes puedan reproducir emociones intensas que han experimentado en el pasado, por ejemplo, planteando preguntas relativas al "peligro de muerte". Los relatos aportados como respuesta a esta pregunta, según este autor, presentan casi siempre un cambio de estilo respecto al discurso más cuidado aproximándose al más vernáculo.

2 Citando a Labov, Moreno Fernández (2012:181-182) señala: "El vernáculo, en el que se presta al discurso propio una atención mínima, aporta los datos más sistemáticos por el análisis lingüístico. El vernáculo se define como la forma de hablar adquirida durante la pre-adolescencia. Su carácter altamente regular se ha observado empíricamente. [...] Todo hablante dispone de una forma vernácula al menos en una lengua, que puede ser un dialecto de prestigio [...] o una variedad no estándar. En algunos casos pueden obtenerse datos sistemáticos de estilos de habla más formales, pero no estaremos seguros de ello hasta que no se hayan contrastado con el vernáculo". 


\subsection{LA ENTREVISTA EMPLEADA EN ESECH}

En relación con la elección de la entrevista como instrumento de recolección de datos, cabe destacar que, en el caso de la perspectiva sociolingüística, las investigaciones sobre lenguaje espontáneo se han desarrollado empleando entrevistas semiestructuradas, donde el entrevistador constituye un público ideal, pues es atento, interesado y receptivo, según Labov (1983). A pesar de que, en cierta medida, la situación comunicativa no es completamente natural, ya que la conversación gira en torno a un conjunto de preguntas formuladas por el entrevistador, este autor destaca que este tipo de entrevistas es muy cercano a la conversación espontánea, si los entrevistadores se proponen superar las constricciones propias del formato de la entrevista. Asimismo, su naturaleza semiestructurada entrega ventajas al investigador que una conversación absolutamente libre no proporcionaría, debido a que su carácter fragmentario podría requerir un enfoque analítico diferente.

Considerando la importancia que, en el marco del enfoque sociolingüístico laboviano, se le atribuye a la obtención de muestras lingüísticas representativas del habla natural de los informantes, las entrevistas han desempeñado un papel esencial en tanto instrumento de recolección de datos. En este sentido, siguiendo a Moreno Fernández (2012) enfatizamos que

la entrevista sociolingüística se ha revelado y manejado como la principal fuente para la provisión de materiales analizables desde una perspectiva cuantitativa variacionista $y$, por tal motivo, muchas de sus dimensiones han recibido la debida atención en diferentes estudios, dimensiones como su valor estilístico, su representatividad en relación con el vernáculo, su contenido temático o su dinámica discursiva (duración, contexto, situación, características de los interlocutores) (Moreno Fernández, 2012: 179).

De acuerdo con el autor recién citado, es la entrevista semidirigida el medio más adecuado para obtener muestras de lengua hablada en la cantidad y con la calidad necesarias para su análisis cuantitativo y, asimismo, es el medio más adecuado para registrar sistemáticamente el habla vernácula, en la que se presta una atención mínima al discurso propio (Moreno Fernández, 2012: 180). Esto implica, por supuesto, no olvidar el efecto de la paradoja del observador. Para los fines de esta investigación y para la superación de la mencionada paradoja, la metodología utilizada en la realización de las entrevistas se ajustó a los siguientes criterios:

1. Se procuró generar un clima de confianza con el objetivo de obtener el estilo de habla más espontáneo del sujeto. Por ello, no se 
descartó la posibilidad de realizar la entrevista a personas cercanas al entrevistador, por ejemplo, familiares o amigos, resguardando, en todos los casos, la congruencia de estatus de los sujetos.

2. Idealmente, los hablantes debían olvidar que la entrevista estaba siendo grabada, es decir, su comportamiento debía ser lo más natural posible. Para ello, el entrevistador debía emplear los pronombres de tratamiento que utilizaba normalmente con el informante.

3. Para conseguir el estilo vernáculo esperado, se procuró que la entrevista se realizara en un lugar tranquilo, idealmente, en la casa del informante o en un lugar que no le fuera extraño, a fin de garantizar su comodidad. Asimismo, se optó por lugares sin ruido ambiental.

Junto con lo anterior, se les entregó a los informantes una carta de consentimiento informado (ver Anexo), elaborada por el equipo del ESECH, a fin de que los hablantes contaran con la información y se sintieran con la confianza que son necesarias para llevar a cabo la entrevista ${ }^{3}$.

Cabe señalar, por otro lado, que el cuestionario solo constituyó una guía para el entrevistador que le permitiera llevar a cabo la conversación de acuerdo con ciertas secciones. El orden de estas, así como las preguntas obligatorias, debía ser respetado. No obstante, el formato de la entrevista contemplaba preguntas optativas que se realizaron atendiendo a las características del informante. En cuanto a su estructura, el cuestionario comprendía las siguientes secciones:

1. Datos sociodemográficos: preguntas destinadas a obtener la información sociodemográfica del entrevistado, para su posterior estratificación.

2. Narración de experiencias personales: preguntas destinadas a obtener relatos de experiencias significativas para el informante (anécdotas, sustos, accidentes, enfermedades, etc.).

3. Temas misceláneos y preguntas de opinión: en esta sección se incluyeron preguntas acerca de variados temas, como tiempo/clima, familia, amistad, costumbres, lugar de residencia o preguntas de opinión. Asimismo, se formularon preguntas específicas considerando los diferentes sexos, grupos etarios y niveles socioeconómicos de los

\footnotetext{
Dicha carta de consentimiento fue visada por el Comité de Ética de la Investigación en Ciencias Sociales y Humanidades de la Facultad de Filosofía y Humanidades de la Universidad de Chile.
} 
entrevistados. Cabe señalar que las secciones 2 y 3 del cuestionario eran las destinadas a generar la mayor cantidad de discurso natural.

4. Lectura de texto: sección cuya finalidad era obtener una muestra de estilo de habla formal del entrevistado.

5. Lectura de listado de palabras: en esta sección del cuestionario se pretendía que el sujeto leyera un listado de palabras, incluidos algunos distractores, a fin de que profiriera las eventuales variables fonológicas de la comunidad lingüística en estudio.

6. Preguntas sobre actitudes lingüísticas: preguntas destinadas a recopilar información sobre el grado de conciencia del entrevistado acerca de su propia actuación lingüística o de la actuación lingüística de otros grupos de la comunidad de habla en estudio.

El conjunto de antecedentes antes proporcionado nos permitió seleccionar, entre un conjunto amplio de entrevistas, las que conformarían el corpus del ESECH. Los criterios para dicha selección son los que siguen:

a) Cumplimiento de la duración mínima de la entrevista (50 minutos)

b) Ajuste al formato de la entrevista

a. Secciones del cuestionario

b. Carácter secuencial de la formulación de las preguntas de las distintas secciones

c. Espontaneidad en la formulación de las preguntas

d. Calidad y formato de la versión electrónica del audio

e. Versión electrónica e impresa del documento transcrito

f. Denominación de los archivos electrónicos (audio y texto transcrito), según la codificación solicitada

c) Calidad del sonido

d) Cantidad natural de corpus obtenido de parte del informante (longitud de los turnos de habla del sujeto entrevistado)

e) Grado de naturalidad del habla del sujeto entrevistado

f) Ajuste a las convenciones estipuladas previamente a la transcripción y etiquetado de texto ${ }^{4}$

4 Para la transcripción de las entrevistas se emplea ortografía convencional, incluidos los acentos gráficos. Asimismo, se utiliza un sistema de etiquetas de apertura y cierre del tipo $<$ etiqueta $>$ texto </etiqueta $>$, de acuerdo con el Standard Generalized Markup Language (SGML) contenido en el Text Encoding Initiative (TEI). Dicho sistema permite el intercambio 
a. Ajuste a los signos, marcas y etiquetas requeridas

b. Ajuste a los criterios de ortografía convencional requeridos

\subsection{Procedimiento de estratificación social utilizado en ESECH}

Para la estratificación de los sujetos que conforman el corpus, se empleó el sistema de adscripción de estatus social empleado en ESECH que parte de una asignación de puntaje según las siguientes variables: 1) nivel educacional, 2) profesión u ocupación y 3) comuna de residencia, cada una de las cuales considera una puntuación entre 1 y 7 , correspondiente a siete niveles en cada caso, como se especificará más adelante. A su vez, a cada variable se le otorgó una ponderación distinta, a saber, 3 puntos para el nivel educacional, 2 para la variable profesión u ocupación y 1 punto para la variable comuna de residencia. Por consiguiente, el puntaje final de estratificación de los sujetos resulta de la suma de los puntajes parciales obtenidos en cada variable, el que se calcula multiplicando la ponderación antes señalada con la puntuación correspondiente. La explicación de la selección de dichas variables se encuentra en el estudio de Prieto (1995-1996), el que fue perfeccionado y actualizado con base en los estudios acerca de la realidad socioeconómica chilena de la empresa de Investigaciones de Mercado y Opinión Pública (ADIMARK, 2003), la Descripción Básica de los Niveles Sociales realizada por el Instituto Consultor en Comercialización y Mercado (ICCOM, 2005) y la propuesta de estratificación de la Asociación de Investigadores de Mercado (AIM, 2008). Cabe precisar que el modelo de nivel socioeconómico fue adaptado de estos estudios de mercado en función de los objetivos que se propone el grupo ESECH, que busca definir un perfil más bien sociocultural de la comunidad lingüística en estudio. En consideración a esto, para la estratificación socioeconómica, una de las variables más determinantes fue el nivel educacional y, por esta razón, es la que asigna el mayor puntaje. Asimismo, el nivel de ingresos demuestra muy poca relevancia para los estudios de mercado antes mencionados, por ello no fue considerado en la determinación de los perfiles socioeconómicos. La mencionada escala incluye cuatro estratos socioeconómicos, a saber: bajo, medio bajo, medio y

de datos entre investigadores y es el mismo que sugiere emplear el Proyecto para el Estudio Sociolingüístico del Español de España y América (PRESEEA), del cual formamos parte. Entre las etiquetas que se usan están las que siguen: etiquetas de documentación, convenciones sobre la ortografía y puntuación, etiquetas de ruido, etiquetas para aspectos fónicos, etiquetas léxicas, etiquetas de dinámica discursiva, etiquetas de lengua y etiquetas de transcripción. 
medio alto, para cuya diferencia se cauteló la consistencia entre las variables antes mencionadas de manera que respondieran a lo que Prieto (1995-1996), siguiendo a Lenski (1954) denominara como congruencia de estatus ${ }^{5}$.

De la asignación de los mencionados puntajes se distinguieron cuatro grupos socioeconómicos ${ }^{6}$, definidos según cuatro rangos:

1. Medio alto (MA): $42-36$

2. Medio (M): $35-27$

3. Medio bajo (MB): $26-18$

4. Bajo (B): $17-6$

El intervalo de cada rango responde a la mayor coincidencia con los puntajes que han establecido los estudios sociológicos y de mercado para la clasificación de estratos socioeconómicos, aunque con las debidas adaptaciones, puesto que en esta muestra no se incluyen ni el grupo de la extrema pobreza ni el de la extrema riqueza, debido a las dificultades para acceder a este tipo de informantes. Por consiguiente, los intervalos propuestos corresponden a un cálculo con una forma piramidal, donde se resta la diferencia entre cada rango de puntaje: $42-36=6$ (MA), 35-27 $=8$ (M), $26-18=8(\mathrm{MB})$ y $17-6=11(\mathrm{~B})$.

A continuación, especificaremos las variables utilizadas para la adscripción de estatus en el ESECH. Como ya señalamos, en cada variable se contempla una puntuación entre 1 y 7 , dependiendo del nivel que corresponda, la que se multiplica por la ponderación de la variable, antes indicada.

5 Lenski (1954) acuñó el término cristalización o congruencia de estatus. Se considera que un individuo es congruente con su estatus cuando las puntuaciones obtenidas en las diferentes dimensiones usadas para medir el estatus son más o menos iguales, independiente de que sus rangos sean altos, bajos o estén en una extensión media. Cuando las puntuaciones del individuo son muy diferentes, se habla de incongruencia de estatus.

6 En la actualidad, el concepto de clase social ha sido relativizado, puesto que se basa en la estructura social proveniente del capitalismo industrial del siglo XIX. Consecuentemente, se han planteado nuevas consideraciones que incorporan dimensiones de estratificación, las que emplean otros factores sociales como la ocupación, el estatus, el poder y aquellos que pudieran ser significativos para la clasificación socioeconómica del sujeto. Siguiendo las propuestas realizadas en el ámbito hispánico, en el ESECH preferiremos el término grupo socioeconómico, pues este último implica tanto el nivel económico como el nivel cultural de los sujetos. 


\subsubsection{Variable nivel educacional}

La escala de rangos utilizada para la clasificación de los sujetos de la muestra, en relación con esta variable, fue la siguiente:

1. Básica Incompleta

2. Básica Completa

3. Media Incompleta

4. Media Completa / Media Técnica Profesional Incompleta

5. Media Técnica Profesional Completa / Técnica Profesional Incompleta

6. Técnica Profesional Completa / Universitaria Incompleta

7. Universitaria Completa

\subsubsection{Variable profesión u ocupación}

A diferencia de lo que plantea el modelo de nivel socioeconómico propuesto por los estudios de mercado, esta variable resulta determinante para el estudio de fenómenos lingüísticos. Así, se elaboró la siguiente escala de rangos en la clasificación de los sujetos:

1. Desempleado (nunca ha trabajado o busca trabajo por primera vez) Cesante

2. Obrero no calificado

Trabajador por cuenta propia no técnico ni profesional (jardinero, pintor, lustrabotas, gásfiter, lavandera, etc.)

Servicio doméstico (mozo, chofer, empleada doméstico)

Empleado público (grados 27 a 31)

3. Obrero calificado (tornero, mecánico, técnico de automóviles, etc.) Capataz, jefe de sección industrial

Propietario de un pequeño negocio (taller, pequeño almacén, quiosco, etc.)

Empleado público (grados 21 a 26)

Estudiante jefe de hogar

Chofer de taxi o de camiones

4. Empleado administrativo de baja categoría (hasta jefe de sección) Vendedores de productos de empresas grandes

Empleados públicos (grados 16 a 20, profesionales en su mayoría)

Profesores primarios o de educación general básica 
5. Empleado administrativo de alta categoría desde Jefe de Departamento (ejecutivos de bajo rango)

Propietarios de negocios medianos

Empleados públicos (grados 8 a 15, profesionales)

Dueños de taxi (con 2 o más taxis que no conducen)

Profesores secundarios

6. Mediano empresario

Ejecutivo joven

Propietarios de negocios grandes

Profesionales (trayectoria de 10 o 20 años)

Empleados públicos (grados 4 a 7)

Profesores universitarios

7. Gran empresario

Altos cargos en grandes empresas

Profesional liberal de éxito que obtenga renta principalmente del ejercicio de su profesión (abogado, médico, ingeniero, etc.)

Empleados públicos (grados 1, 2, 3, ministros, subsecretarios)

\subsubsection{Variable comuna de residencia}

La selección de la variable comuna residencia, en el marco de trabajo del grupo ESECH, responde al hecho de que tanto Prieto (1995-1996) como ICCOM (2005) le asignan relevancia como factor segregador. De este modo, las comunas se distribuyeron en siete estratos, en orden ascendente de nivel socioeconómico, como sigue:

1. La Pintana, Cerro Navia, Lo Espejo, Renca, San Ramón.

2. La Granja, Lo Prado, Pedro Aguirre Cerda, Conchalí, El Bosque, Pudahuel, Recoleta.

3. Quinta Normal, San Joaquín, San Bernardo, Puente Alto, Cerrillos.

4. Quilicura, Estación Central, Independencia, Maipú, La Cisterna.

5. Santiago, Huechuraba, Peñalolén, La Florida, Macul, San Miguel.

6. La Reina, Providencia, Ñuñoa.

7. Vitacura, Las Condes, Lo Barnechea. 


\subsection{Población y MUESTRA}

En nuestra investigación se considerará la población constituida por hombres y mujeres de la Región Metropolitana de más de 20 años de edad. Tomando en consideración que tanto la segmentación de la comunidad de habla en grupos socioeconómicos como la división entre hombres y mujeres quedó ya explicada, cabe señalar que los tres grupos etarios que hemos considerado apelan a tres momentos vitales (Blas Arroyo 2005), es decir, en el primer grupo (20 a 34 años) se encuentran los sujetos que ingresan al mundo laboral o que llevan poco tiempo desempeñando una profesión o actividad específicas, pues a partir de ese momento hay un desarrollo de los patrones "adultos" de variación. Por su parte, en el segundo grupo (35 a 54 años) están incluidos los sujetos que tienen un desarrollo laboral pleno y, finalmente, en el tercer grupo etario (55 años y más) se hallan los sujetos que están prontos a dejar el mundo laboral o que ya lo han dejado. En este sentido, se trabaja con etapas por las que pasa el hablante, que suelen estar vinculadas con la cultura de cada comunidad lingüística.

El cuestionario se aplicó a una muestra del tipo "muestra por cuotas con afijación uniforme", en la que se divide a la población en estratos o categorías y se asigna una cuota a cada uno de los distintos estratos (López Morales 1994: 58). Por lo que respecta al tamaño de la muestra, hemos optado por conformarla con un número de informantes considerado, en general, como suficiente en la bibliografía sociolingüística (cf. Trudgill y Hernández Campoy 2007, s. v. representatividad ${ }^{7}$ y López Morales 1994: 52). La muestra, así conformada, comprende un total de 192 entrevistas realizadas a igual número de sujetos, distribuidos como se indica en la tabla 1 que mostramos a continuación:

\footnotetext{
En general, se sugiere que cinco informantes por celda es suficiente. Sin embargo, lo esencial es garantizar que la selección de los informantes cubra todos los aspectos sociodemográficos implicados en la investigación. En nuestro caso, al no existir datos censales provenientes de organismos oficiales nacionales acerca del número de lo que nosotros hemos denominado "hablante santiaguino", hemos optado por conformar una muestra con un número importante de informantes de acuerdo con la bibliografía que en sociolingüística alude al tema, donde, en general, se habla de entre 50 y 150 sujetos. Además, nos basamos en que es la complejidad interna de cada universo la que, finalmente, determina el tamaño muestral.
} 
Tabla 1. Proporción de sujetos según características sociodemográficas de sexo, edad y grupo socioeconómico

\begin{tabular}{|l|c|c|c|c|c|c|c|}
\hline \multirow{2}{*}{$\begin{array}{l}\text { Grupo } \\
\text { socioeconómico }\end{array}$} & \multicolumn{2}{|c|}{$20-34$} & \multicolumn{2}{c|}{$35-54$} & \multicolumn{2}{c|}{55 y más } & \\
\cline { 2 - 9 } & $\mathrm{H}$ & $\mathrm{M}$ & $\mathrm{H}$ & $\mathrm{M}$ & $\mathrm{H}$ & $\mathrm{M}$ & Total \\
\hline Medio alto & 8 & 8 & 8 & 8 & 8 & 8 & $=48$ \\
\hline Medio & 8 & 8 & 8 & 8 & 8 & 8 & $=48$ \\
\hline Medio bajo & 8 & 8 & 8 & 8 & 8 & 8 & $=48$ \\
\hline Bajo & 8 & 8 & 8 & 8 & 8 & 8 & $=48$ \\
\hline & 32 & 32 & 32 & 32 & 32 & 32 & $=192$ \\
\hline
\end{tabular}

En la tabla 2, por su parte, se presentan las características sociodemográficas de los integrantes de la muestra, clasificados de acuerdo con su sexo, edad y grupo socioeconómico. 

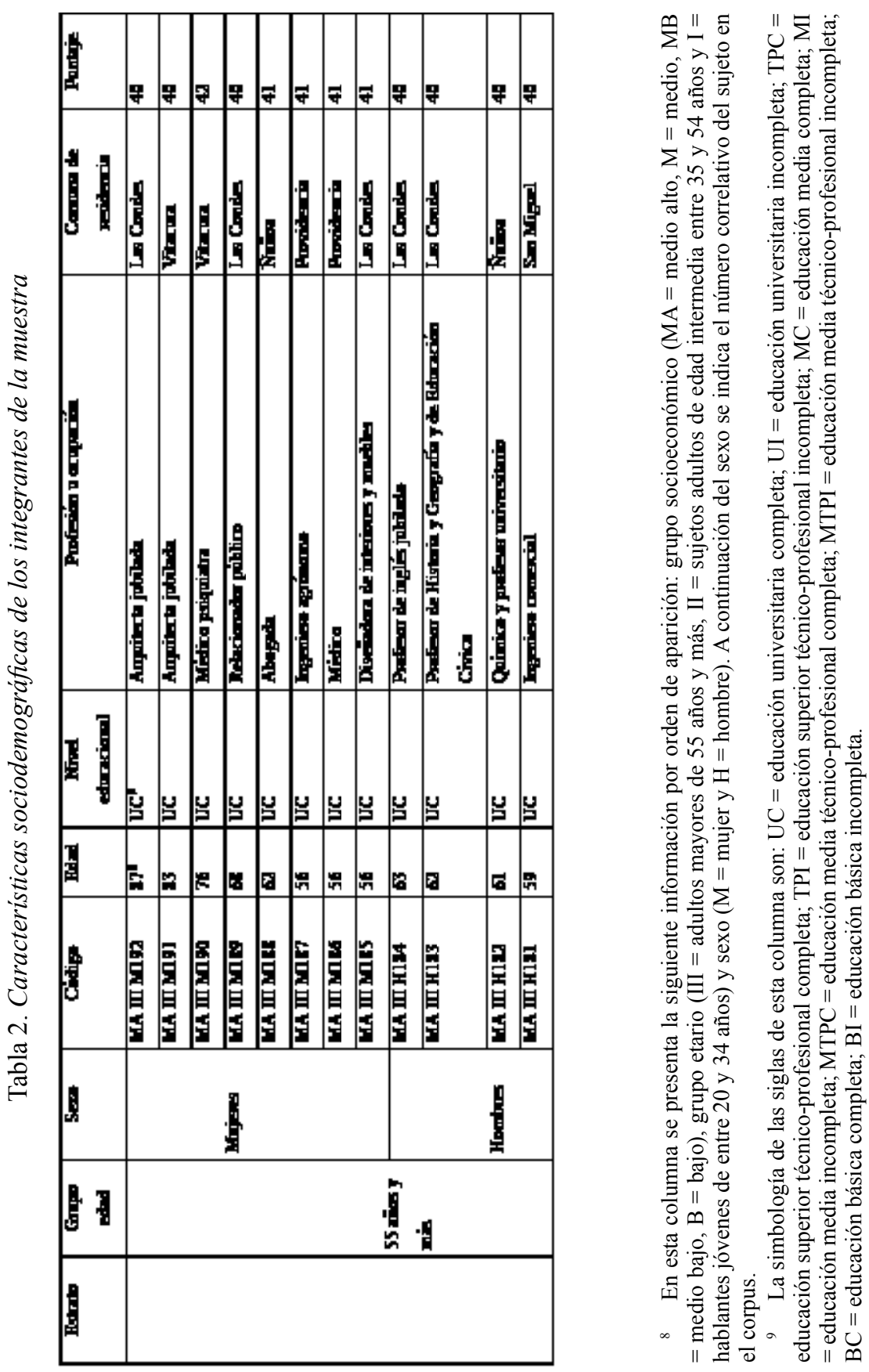


\begin{tabular}{|c|c|c|c|c|c|c|c|c|c|c|c|c|c|c|c|c|c|c|c|}
\hline F & ช & $\exists$ & $g$ & $\stackrel{9}{9}$ & $g$ & $F$ & 案 & $F$ & $g$ & $F$ & $F$ & $F$ & 웅 & F & 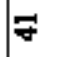 & 웜 & \% & $\neq$ & $F$ \\
\hline 置 & 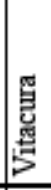 & 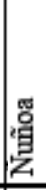 & 言 & $\begin{array}{l}8 \\
\frac{8}{8} \\
8 \\
8 \\
6 \\
6 \\
0\end{array}$ & 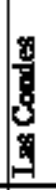 & 惫 & 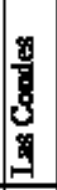 & 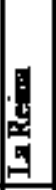 & 篦 & 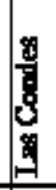 & 量 & 望 & 标 & 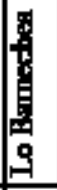 & 量 & 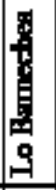 & 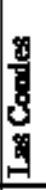 & 置 & 置 \\
\hline 吾 & 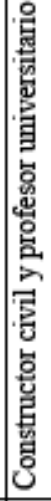 & 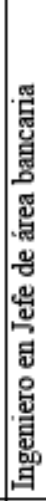 & 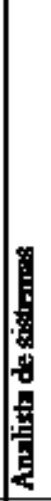 & 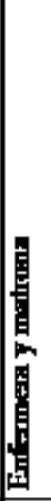 & 焉 & 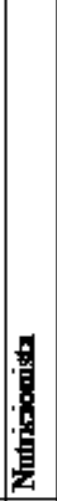 & 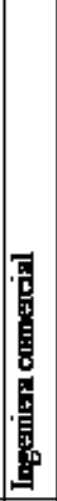 & 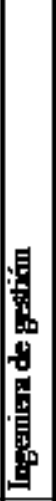 & 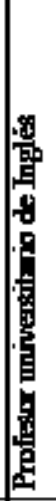 & 咅 & 道 & 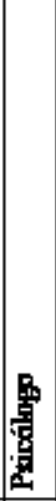 & 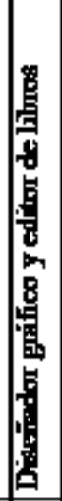 & 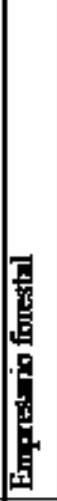 & 总 & 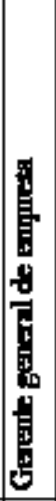 & 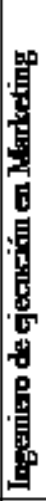 & 商 & 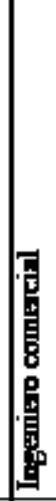 \\
\hline 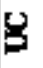 & יִ & יִ & $\beta$ & $\beta$ & $\beta$ & $\beta$ & $\beta$ & 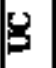 & $\beta$ & 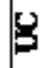 & 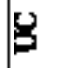 & $\beta$ & 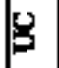 & $\beta$ & 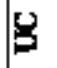 & $\beta$ & 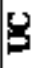 & $\beta$ & $\beta$ \\
\hline R & $\stackrel{n}{\curvearrowleft}$ & $\because$ & $\curvearrowleft$ & इ & 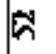 & $\hbar$ & $\bar{\kappa}$ & $R$ & 9 & 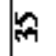 & $\approx$ & 至 & ద스 & of & g & o & $F$ & go & 果 \\
\hline \multirow[t]{4}{*}{ 总 } & 贫 & 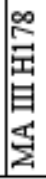 & 点 & 㧹 & 夆 & 总 & 点 & 象 & 苞 & 国 & 总 & 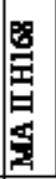 & 原 & 道 & 总 & 总 & 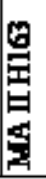 & 国 & 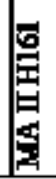 \\
\hline & & & & & & & & & 量 & & & & & & 算 & & & & \\
\hline & & & & & & & & & & & 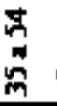 & & & & & & & & \\
\hline & & & & & & & & & 愛 & 0 & & & & & & & & & \\
\hline
\end{tabular}




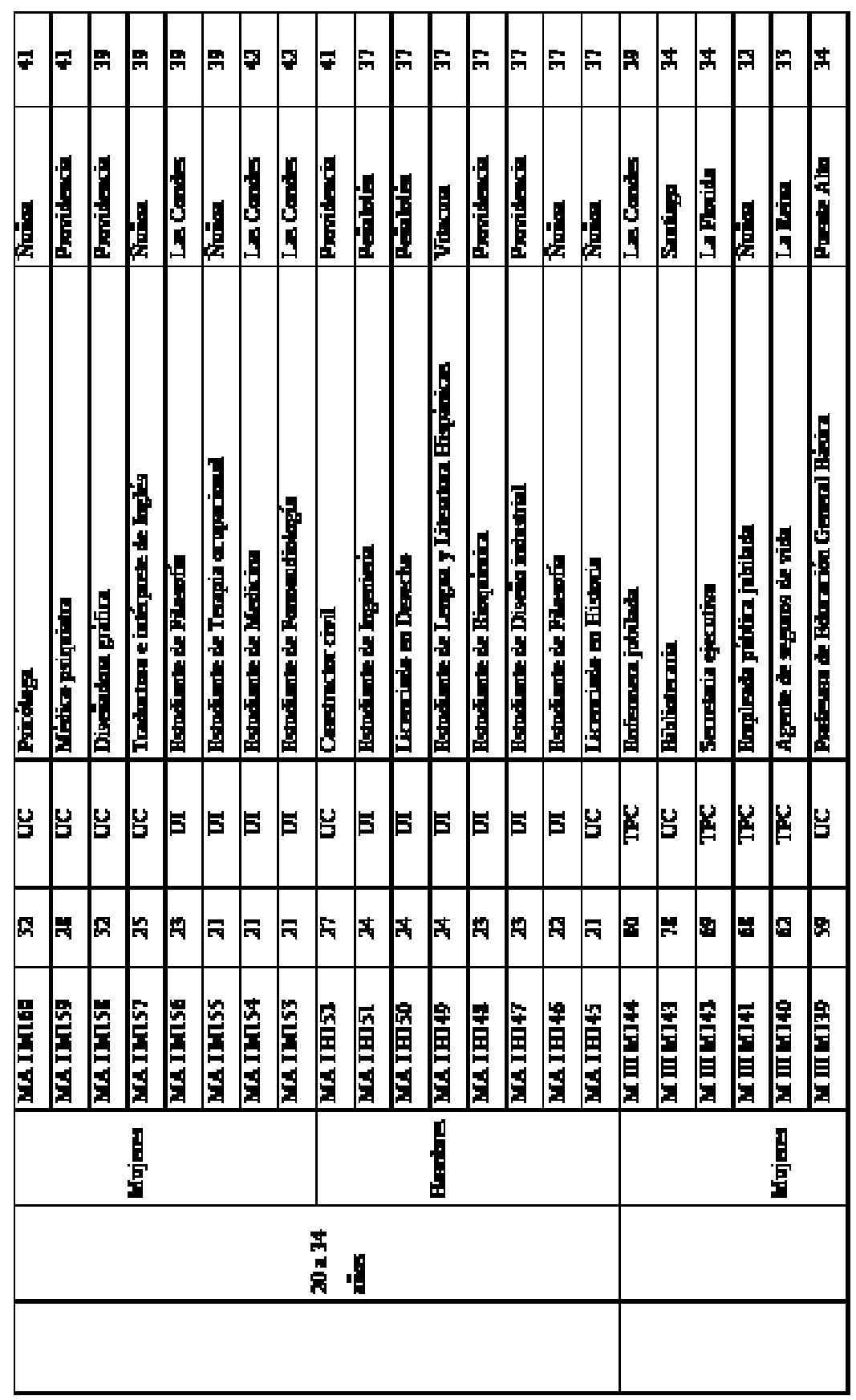




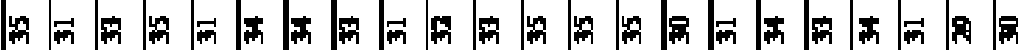

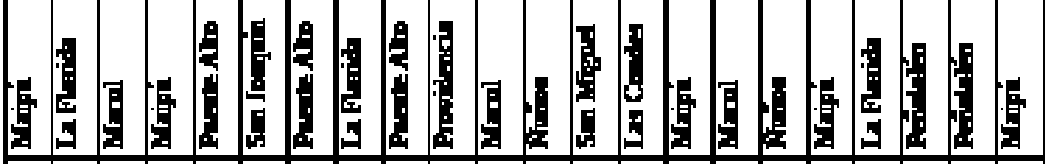

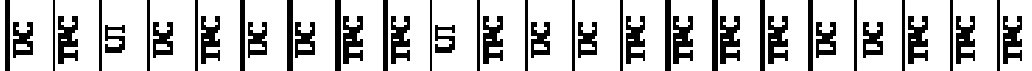

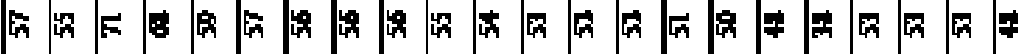

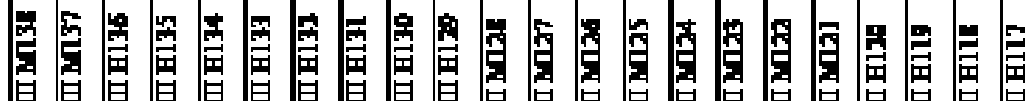

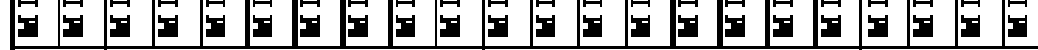

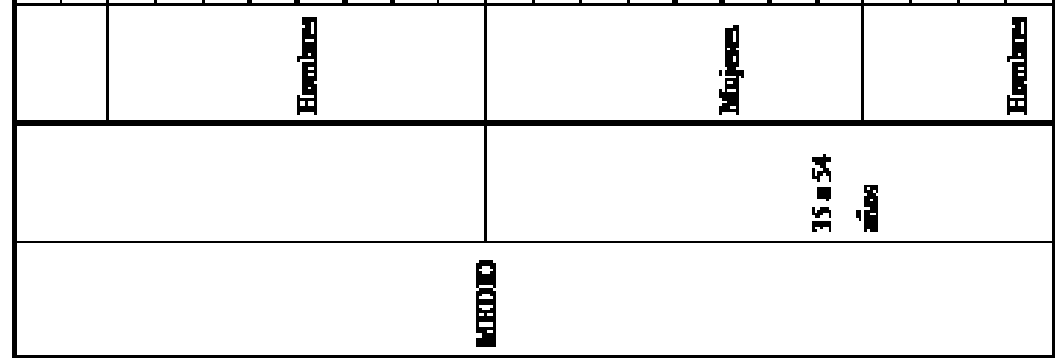




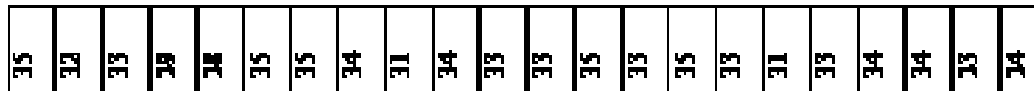

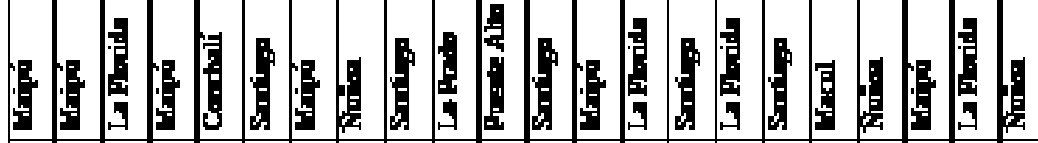

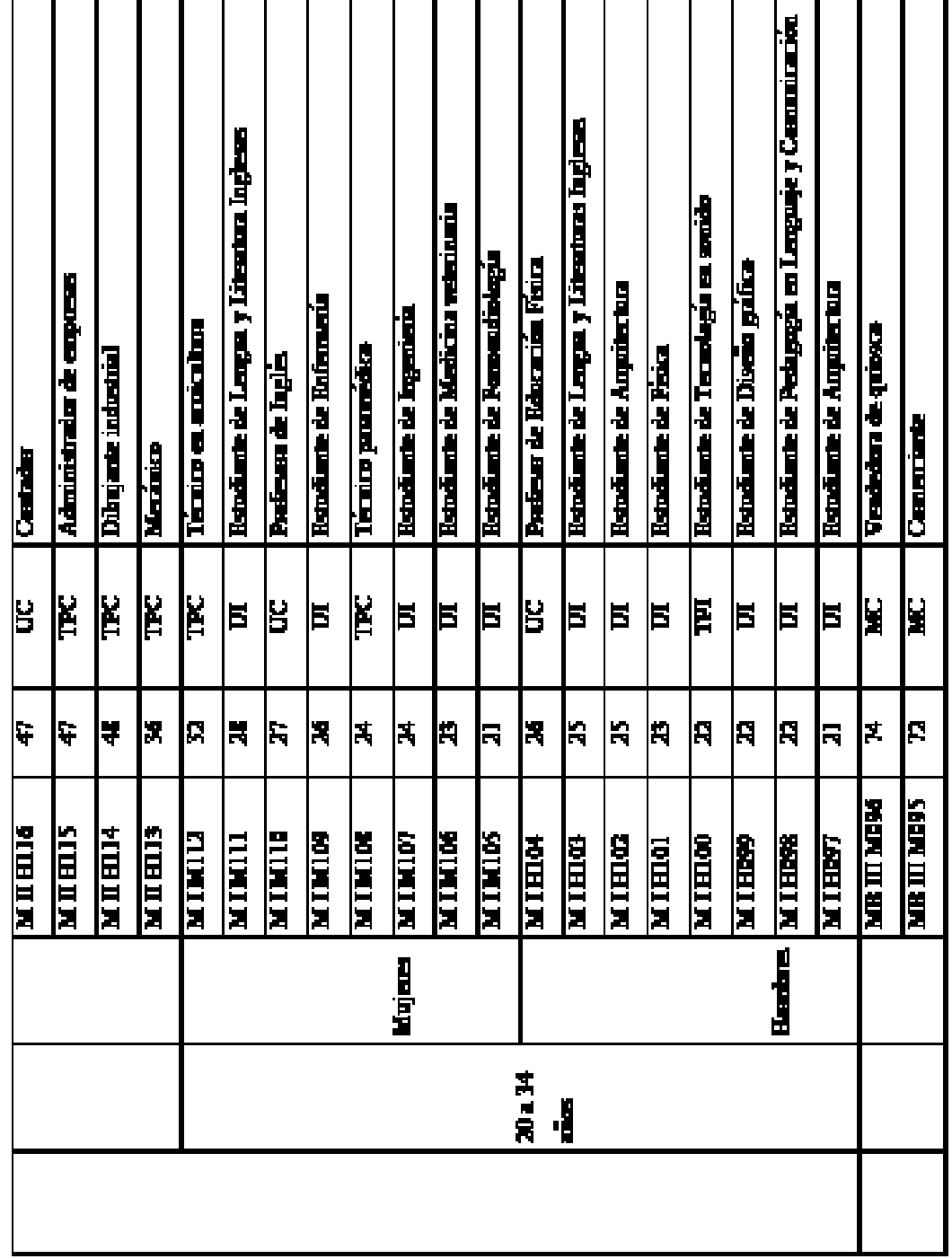




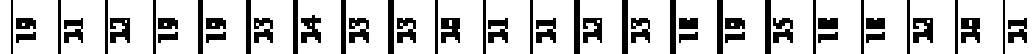

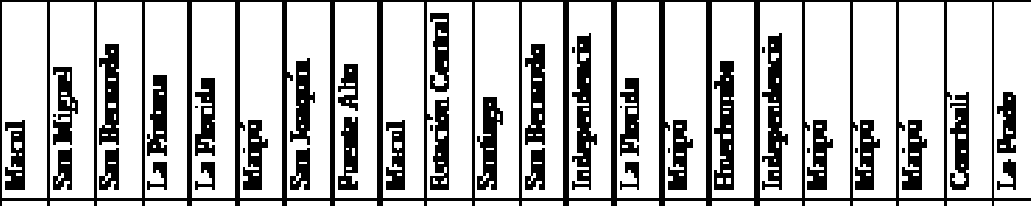

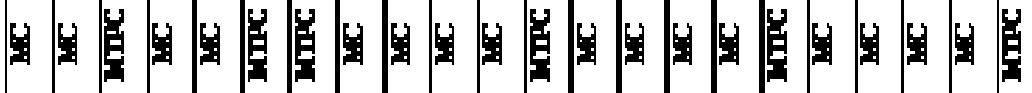

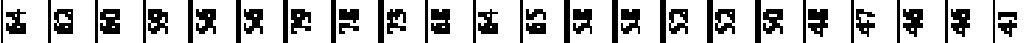

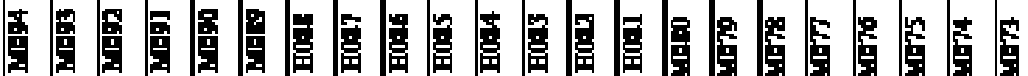

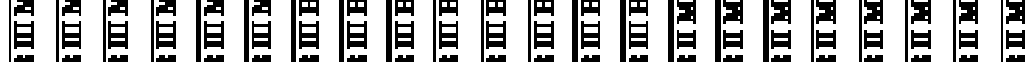

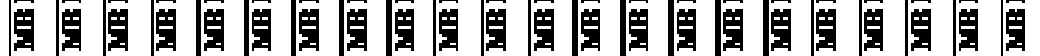

\begin{tabular}{|c|c|c|}
\hline 量 & 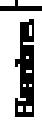 & 具 \\
\hline & & 藻 \\
\hline
\end{tabular}




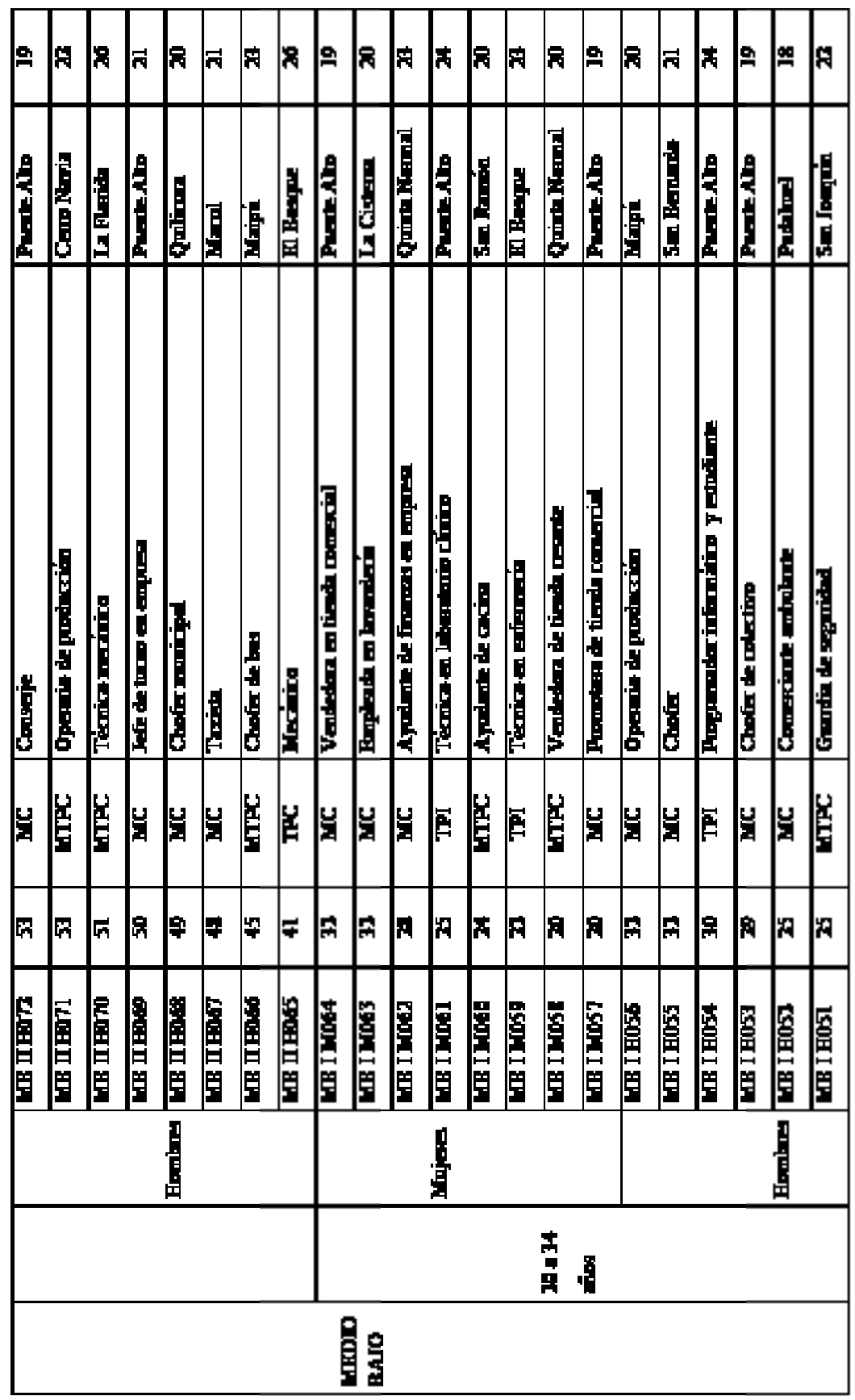




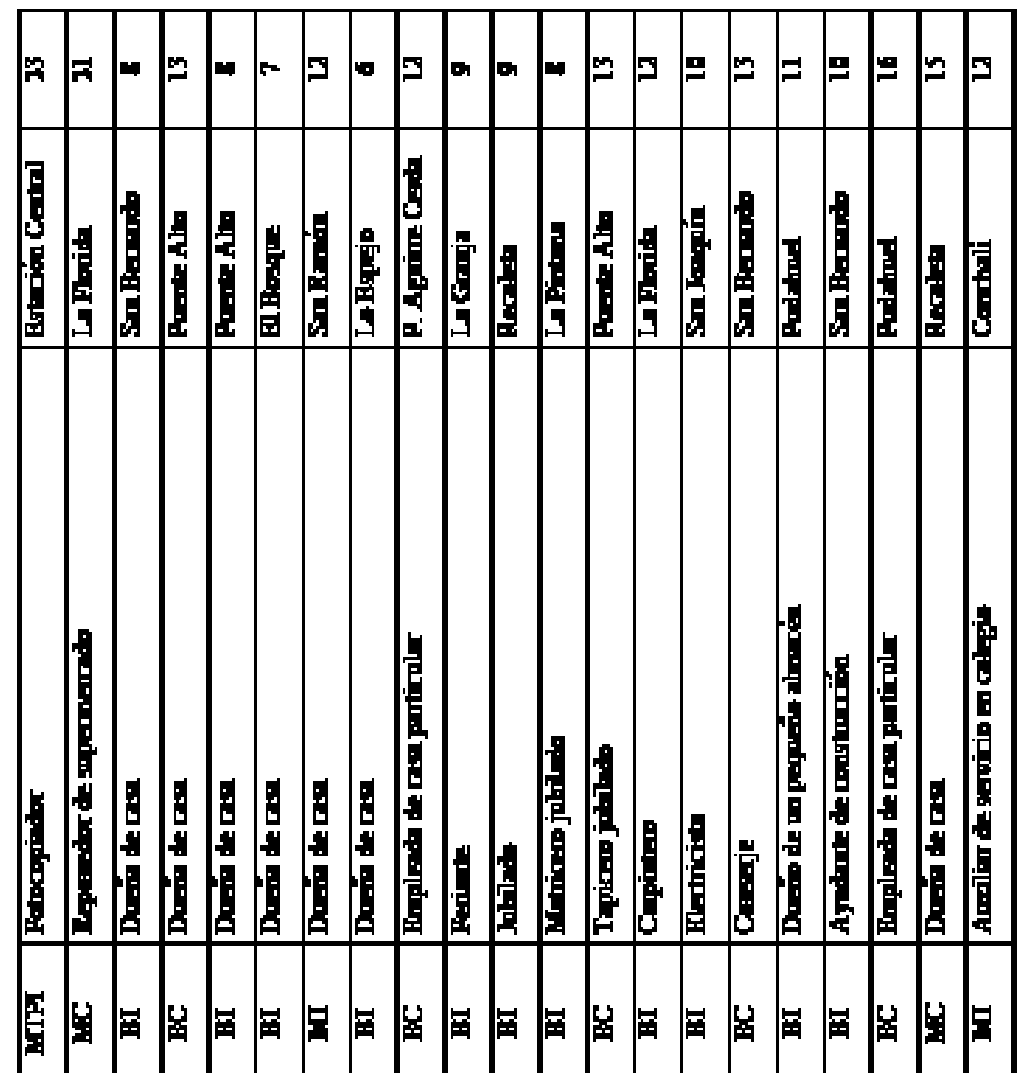

舟居 为

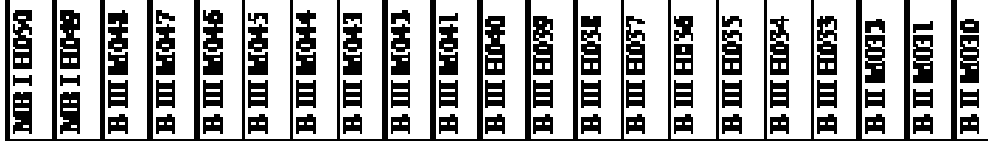

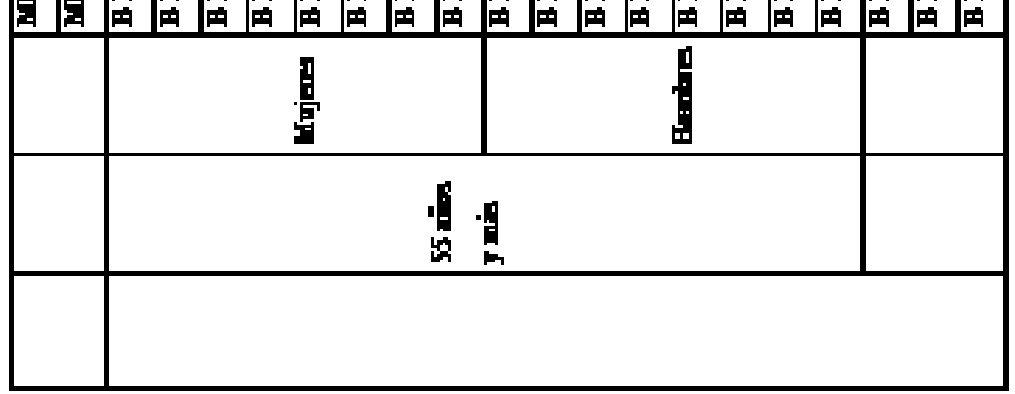




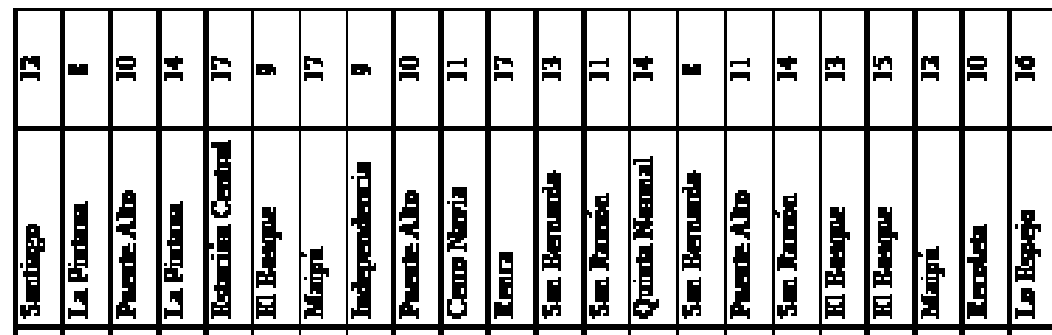

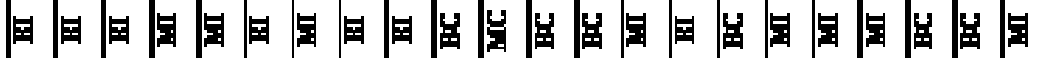

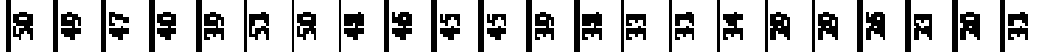

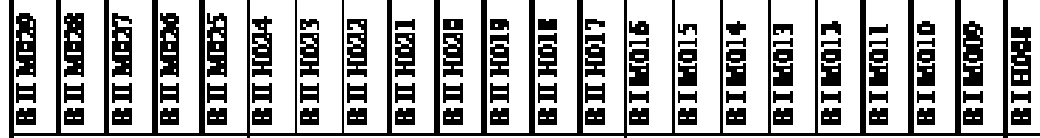

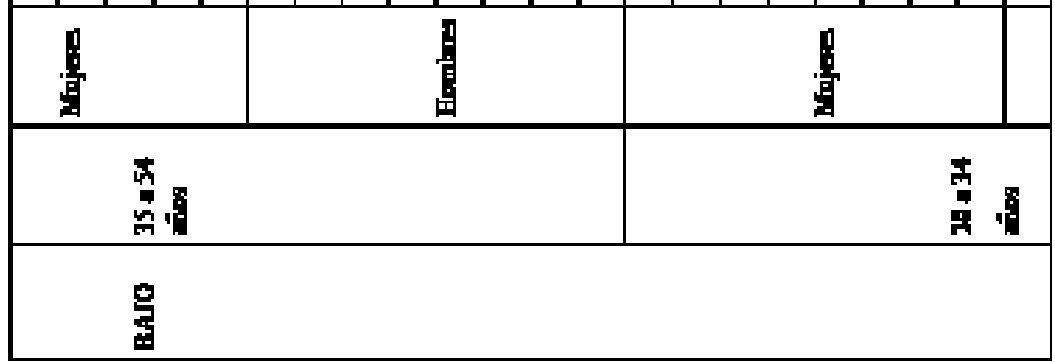




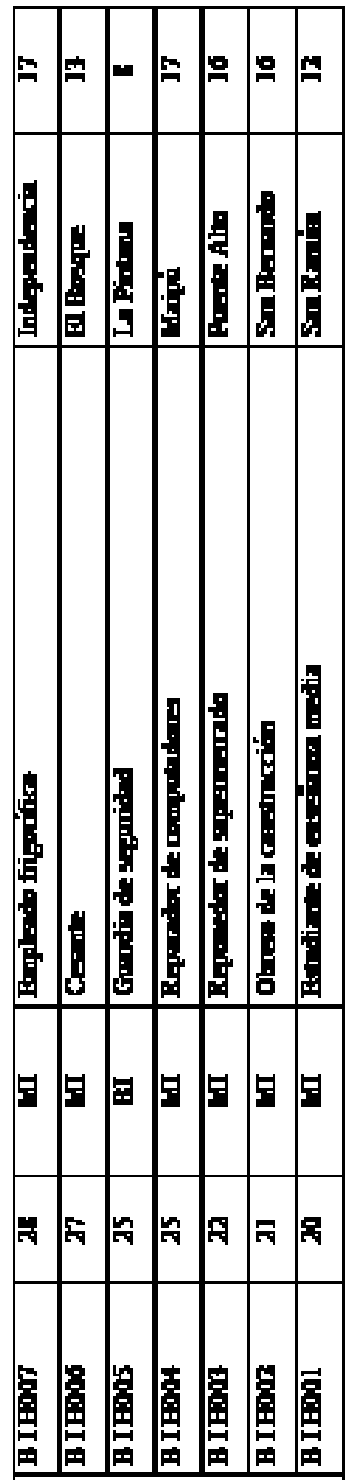




\section{CONCLUSIÓN Y PROYECCIONES}

Los datos obtenidos por el ESECH han permitido llevar a cabo varias investigaciones que contribuyen a describir la variación sociolingüística en el español hablado en Santiago. Entre ellas, algunas tesis de grado que se han centrado en el estudio de los marcadores del discurso, en tanto fenómeno variable, específicamente, en el análisis pragmático y sociolingüístico de los marcadores discursivos de reformulación (Rojas y Rubio 2012) y de los marcadores argumentativos (Castro et al. 2013). Otros trabajos se han dedicado al estudio de fenómenos de naturaleza sintáctica, como el dequeísmo (Zabala 2012 y San Martín 2014). En esta última tesis, también se incluye el estudio del queísmo y de aspectos variables a nivel discursivo como el discurso referido y los marcadores de reformulación. En otro orden, se han desarrollado investigaciones cuyo foco han sido las narraciones de experiencia personal (Guerrero 2009 y 2014), que no solo se han basado en el corpus del ESECH, sino que también han tomado datos del macrocorpus PRESEEA para llevar a cabo estudios contrastivos (Olave y Prado 2014). Junto con lo anterior, los miembros del ESECH han publicado un buen número de estudios que dan cuenta de investigaciones que permiten describir, en especial, los niveles sintáctico y pragmático-discursivo de la variación sociolingüística del español de Santiago de Chile. En los últimos años, además, el equipo ha incorporado herramientas estadísticas que permiten verificar la existencia de correlaciones entre los factores sociales y los lingüísticos.

Por otro lado, cabe señalar que los miembros del ESECH se dedican también a la construcción del corpus PRESEEA de Santiago de Chile, que se encuentra hoy en su etapa de edición final ${ }^{10}$. Este corpus, en particular, difiere del ESECH no solo en la cantidad de entrevistas que considera (108), sino que también en que no utiliza un sistema de estratificación como el que aquí hemos descrito, puesto que incluye solo la variable nivel educacional, además del sexo y la edad de los informantes. Ambos corpus, sin embargo, permitirán desarrollar un sinnúmero de investigaciones sobre la variación en todos sus niveles, fonético-fonológico, morfológico,

10 Próximamente, el equipo iniciará la recolección de datos para la elaboración del corpus PRESEEA-Valparaíso. 
sintáctico, léxico y pragmático-discursivo. Es igualmente relevante que el ESECH no solo considera un corpus de entrevistas sociolingüísticas, sino que -además- incluye un sub-corpus para el análisis conversacional. Este último se encuentra todavía en una etapa muy incipiente de elaboración y su desarrollo permitirá comenzar una serie de investigaciones en el marco de la sociolingüística interaccional, estableciendo el vínculo analítico y metodológico entre lo cuantitativo y lo cualitativo. Así también, la entrevista empleada en ESECH contempla una sección de preguntas sobre actitudes lingüísticas, que permitirán realizar estudios sobre la valoración y el grado de conciencia que tienen los hablantes sobre su propia lengua.

En consecuencia, los materiales que hemos descrito en este trabajo se están actualizando y complementando de manera permanente. Su retroalimentación con corpus como el del Proyecto del Habla Culta (Rabanales y Contreras 1979 y 1990) y otros que podamos construir más adelante, posibilitará el desarrollo de estudios diacrónicos y contrastivos acerca de la variación sociolingüística en el español de Santiago de Chile y de otras localidades del país, así como con otras comunidades de habla, especialmente, hispánicas. Finalmente, esperamos que este trabajo, que constituye solo una propuesta acerca de la recogida de materiales y su estratificación para el análisis sociolingüístico, pueda dar luces para que otros investigadores lleven a cabo estudios en cualquier nivel de la variación lingüística. Nuestro objetivo es avanzar en este ámbito de la investigación de la lengua española empleando materiales sociolingüísticos para dar cuenta de la realidad sociocultural de diferentes comunidades de habla.

\section{REFERENCIAS BIBLIOGRÁFICAS}

Adimark (Investigaciones de Mercado y de Opinión Pública). 2003. Mapa socioeconómico de Chile. Nivel socioeconómico de los hogares del país basado en datos del Censo. En línea: http://www.adimark.cl/medios/estudios/mapa_socioeconomico_de_chile.pdf (Consulta: 10/01/2012).

Aim (Asociación de Investigadores de Mercado). 2008. Grupos socioeconómicos 2008. En línea: http://www.anda.cl/estudios/textos/DescripcionGSEChile2008.pdf (Consulta: $10 / 01 / 2012)$.

Blas Arroyo, José Luis. 2005. Sociolingüistica del español. Desarrollos y perspectivas en el estudio de la lengua española en contexto social. Madrid: Cátedra.

Castro, Antonieta, Camila Cordero y Gabriela Olave. 2013. Análisis pragmático y sociolingüístico del empleo de los marcadores argumentativos en el español hablado de Santiago de Chile. Tesis para optar al grado de Licenciado en Lengua y Literatura Hispánica mención Lingüística. Santiago de Chile: Universidad de Chile. 
Guerrero, Silvana. 2009. Análisis sociolingüistico de las diferencias de género en narraciones de experiencias personales en el habla juvenil de Santiago de Chile. Tesis para optar al grado de Magíster en Lingüística mención Lengua Española. Santiago de Chile: Universidad de Chile.

2014. Variación discursiva en narraciones de experiencia personal en el español hablado en Santiago de Chile. Tesis para optar al grado de Doctor en Lingüística. Santiago de Chile: Pontificia Universidad Católica de Chile.

Hernández Campoy, Juan y Manuel Almeida. 2005. Metodología de la investigación sociolingüística. Málaga: Editorial Comares.

Iссом (Instituto Consultor en Comercialización y Mercado). (2005). Descripción Básica de los Niveles Sociales Hogares Urbanos Región Metropolitana. En línea: http:/www.cadem.cl/ v1/files/estadistico/descripcionBasica_GSE_ICCOM_2005.pdf (Consulta: 10/01/2012).

Labov, William. 1983. Modelos sociolingüísticos. Madrid: Cátedra.

Lenski, Gerard. 1954. Status Crystallization: A Non-vertical Dimension of Social Status. American Sociological Review, 19, 405-413.

López Morales, Humberto. 1994. Métodos de investigación lingüiśtica. Salamanca: Ediciones Colegio de España. 2004. Sociolingüistica. Madrid: Gredos.

Moreno Fernández, Francisco. 1990. Metodología sociolingüística. Madrid: Editorial Gredos. 1998. Principios de sociolingüística y sociología del lenguaje. Barcelona: Ariel.

2012. Moreno Fernández, Francisco. 2012. Sociolingüistica cognitiva. Proposiciones, escolios y debates. Madrid/Frankfurt: Iberoamericana/Vervuert.

Olave, Belén y Emanuela Prado. 2014. Análisis sociolingüístico de las diferencias de sexogénero en la construcción de narraciones de experiencia personal en tres comunidades de habla española. Tesis para optar al grado de Licenciado en Lengua y Literatura Hispánica mención Lingüística. Santiago de Chile: Universidad de Chile.

Prieto, Luis. 1995-1996. Análisis sociolingüístico del dequeísmo en el habla de Santiago de Chile. Boletín de Filología, Tomo XXXV, 379 - 452.

Rabanales, Ambrosio y Lidia Contreras (eds.). 1979. El habla culta de Santiago de Chile. Materiales para su estudio, Tomo I. Santiago de Chile: Universidad de Chile.

(eds.). 1990. El habla culta de Santiago de Chile. Materiales para su estudio, Tomo II. Bogotá: Instituto Caro y Cuervo.

Rojas, Cristian y Alejandra Rubio. 2012. Análisis pragmático y sociolingüístico del empleo de los marcadores discursivos de reformulación en el habla de Santiago de Chile. Tesis para optar al grado de Licenciado en Lengua y Literatura Hispánica mención Lingüística. Santiago de Chile: Universidad de Chile.

San Martín, Abelardo. 2014. Variación sintáctica y discursiva en el español hablado en Santiago de Chile. Análisis sociolingüistico del queísmo, el dequeísmo, el discurso referido y los marcadores de reformulación. Tesis para optar al grado de Doctor en Filosofía Sección Filología Hispánica. Valladolid: Universidad de Valladolid.

Serrano, María José. 2011. Sociolingüística. Barcelona: Ediciones del Serbal.

Silva-Corvalán, Carmen. 2001. Sociolingüística y pragmática del español. Washington. Georgetown University Press.

Trudgill, Peter y Juan Hernández Campoy. 2007. Diccionario de sociolingüística. Madrid: Gredos.

ZaBALA, MARÍA José. 2012. Aproximación sociolingüistica al dequeísmo en el español actual de Santiago de Chile. Tesis para optar al grado de Magíster en Lingüística mención Lengua Española. Santiago de Chile: Universidad de Chile. 


\section{ANEXO}

Santiago,

\section{Carta de consentimiento informado}

El Grupo de Estudio Sociolingüístico del Español de Chile (ESECH) del Departamento de Lingüística de la Universidad de Chile lo/la está invitando a participar como informante en los estudios que realizarán sobre la relación entre el lenguaje y la sociedad, en el marco del ESECH y del Proyecto para el Estudio Sociolingüístico del Español de España y América (PRESEEA).

El propósito de esta carta es ayudarlo a tomar la decisión de participar o no.

\section{Objetivo de la investigación}

El proyecto se propone el estudio de diferencias en el lenguaje de las personas que hablan español en Santiago de Chile. Para tal efecto, la actividad principal del proyecto consiste en realizar un conjunto de entrevistas representativas del habla santiaguina. A continuación, se seleccionará una serie de características de pronunciación, gramática, vocabulario y uso del lenguaje, cuya presencia será verificada en las mencionadas entrevistas. Por último, se estudiarán los factores del lenguaje, situacionales y sociales relacionados con el empleo de cada característica, a fin de explicar dichos fenómenos.

\section{Procedimientos}

Su participación es completamente voluntaria y consiste en lo siguiente: dar una entrevista de una hora de duración, en la que deberá narrar historias de experiencia personal, opinar sobre temas de conocimiento público, leer dos textos breves, leer una lista de palabras y opinar sobre su propia forma de hablar. Las entrevistas serán grabadas solo en audio y pasarán a formar parte del material que se analizará.

\section{Confidencialidad}

Los resultados de las investigaciones que se realicen se comunicarán en publicaciones y congresos académicos. Su participación en este proyecto 
será completamente anónima, ya que no se revelará su identidad como participante en ningún momento. Usted podrá conocer los resultados de la investigación cuando estén disponibles. Las entrevistas solo serán utilizadas para estos estudios y analizadas por el investigador responsable y un equipo de colaboradores.

\section{Beneficios, riesgos y derechos}

Su participación como informante en esta etapa de la investigación es voluntaria y no será remunerada. Si usted decide participar en esta investigación tendrá la libertad de omitir preguntas hechas en la entrevista y dejar de participar en cualquier momento. Usted no se beneficiará directamente con este estudio, sin embargo, su participación puede ayudar a saber más sobre el habla santiaguina y nuestra propia identidad como hablantes de español.

\section{Investigador responsable y contactos}

El proyecto es dirigido por Abelardo San Martín Núñez, profesor del Departamento de Lingüística de la Universidad de Chile. En caso de tener alguna consulta, usted podrá contactarse con el director del proyecto al número de teléfono 9787027 o a su correo electrónico asmartin@uchile.cl. Además, si tiene alguna duda sobre sus derechos como participante puede contactar al profesor Raúl Villarroel, Director del Centro de Ética Aplicada de la Facultad de Filosofía y Humanidades de la Universidad de Chile. Su número de teléfono es 9787023 y su correo electrónico rvillarr@uchile.cl.

Si usted está dispuesto a participar en la entrevista, por favor firme donde corresponda.

Nombre y firma participante

Nombre y firma investigador responsable 